\title{
Mechanical Behavior of Various Orthodontic Retraction Springs
}

\author{
Rachman Setiawan, Muhammad Idris \& Tito Dwi Prakasa \\ Faculty of Mechanical and Aerospace Engineering, \\ Institut Teknologi Bandung, Bandung, Indonesia \\ Email: rachmans@edc.ms.itb.ac.id
}

\begin{abstract}
Retraction spring is a type of orthodontic apparatus that is used to move a tooth with respect to another by utilizing its spring back effect. It is made of metallic wire formed to individual orthodontic cases. A specific geometry results in a set of force system, consisting of forces and moments, that provides specific movement effect when it is pre-activated to the adjacent teeth. Currently, orthodontists select its geometry depending on their knowledge and experience. It is based on separate and less-than-comprehensive literatures that not all orthodontists have access to. It may result in inaccuracies in treating individual tooth retraction case. Engineering approach to estimating retraction spring structural behavior is proposed through analytical, numerical and empirical methods. Castigliano method is used as the analytical approach, whilst finite element method is used as the numerical approach. The two simulation approaches were compared to the experiments to obtain the best simulation model. The behavior of the simulation models agree well with those of experiments. Hence, the simulation models were used to simulate a large number of geometries to form database of structural behavior of retraction spring that could be used in the geometry selection by orthodontists.
\end{abstract}

Keywords: Castigliano; Finite element method; Orthodonti; Retraction spring.

\section{$1 \quad$ Introduction}

Orthodontic is a specialty of dentistry that is concerned with the study and treatment of malocclusions (improper teeth arrangement). It may be caused by a result of tooth irregularity ordisproportionate jaw. Retraction spring is a type of orthodontic device used for correcting the tooth position using the reaction force of the pre-tensioned spring. Certain geometry of the spring forms a certain system of forces that allows certain dental movement. With the correct selection, the treatment will take place more accurately and reducing the sideeffect to the patients. In order to do so, an orthodontist selects the geometry of the retraction spring based on his/her knowledge and experience, as well as literatures. Then, a piece of raw orthodontic wire is formed to the geometry manually. The wire can be of Stainless steel (SS), Titanium molybdenum alloy (TMA), or Nickel Titanium alloy (NiTi). Manual selection and production of

Received November $24^{\text {th }}, 2010$, Revised June $23^{\text {rd }}, 2011$, Accepted for publication July $25^{\text {th }}, 2011$.

Copyright @ 2011 Published by LPPM ITB \& PII, ISSN: 1978-3051, DOI: 10.5614/itbj.eng.sci.2011.43.3.5 
the retraction spring described above is regarded as a time-consuming process and could introduce inaccuracies, resulting in less-than-effective treatment to the patient in forms of: insufficient retraction effect due to insufficient retraction force, or on the contrary, damage in tissue due to excessive force, or undesired movement. These, in turn, lengthen the treatment time, or worst, could upset the patient.

Among others, below are the primary characteristics that describe a retraction spring: (1) The moment-to-force ratio $(M / F)$ which determines the center of rotation of the tooth during its movement: $(2)$ the retraction force at yield $(F)$; this represents the greatest force that can be delivered from a retraction spring without permanent deformation. Illustration of the effect of the moment-to-force ratio to the center of rotation hence the tooth movement can be seen in Figure 1 [1], where the bodily, or translational, movement can be obtained by setting the ratio to 9.4 for a specific case of canine tooth. Many retraction spring designs were developed by researchers, for instance T-Loop, as shown in Figure 2, with the illustration of the forces and moment produced when it is activated $\left(F_{x}, F_{y}\right)$ and moments $\left(M_{y}, M_{z}\right)$ [2]. Alternative designs exist, namely B-Loop, L-Loop, U-Loop, as in Figure 3 [3,4].

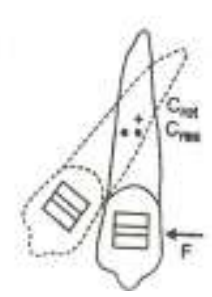

(a) $M / F=0$

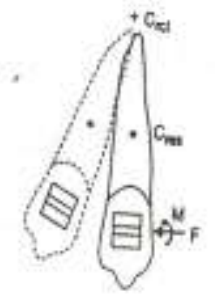

(b) $M / F<9.4$

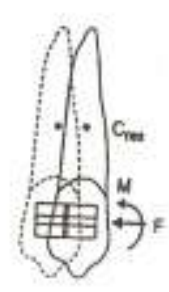

(c) $M / F=9.4$

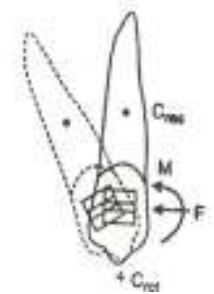

(d) $M / F>9.4$

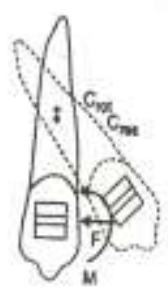

(e) $M / F=\infty$

Figure 1 Effects of retraction process to the tooth movement [1].

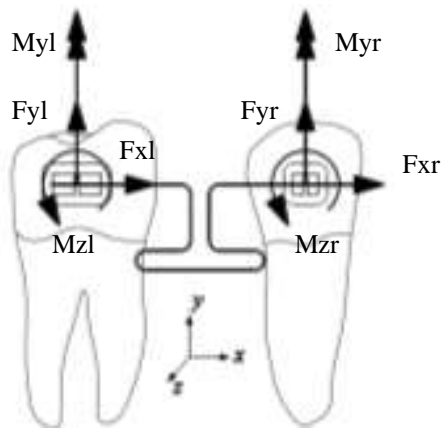

Figure 2 Force system (forces and moments) [2]. 


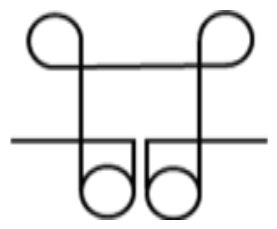

(a) B-loop

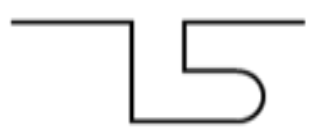

(b) L-loop

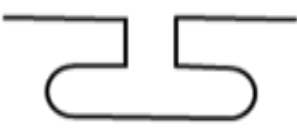

(c) T-loop

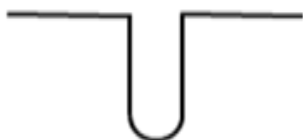

(d) U-loop

Figure 3 Types of retraction springs [4,5].

A number of researchers have described the overview of loop characteristic with respect to the activation distance and the optimum force that can be obtained by a loop design. However, in order to obtain a feasible design, a comprehensive knowledge on the loop characteristics is needed. The orthodontic treatment should consider analytical relations among spring characteristics, design, dimension, and activation distance. Otherwise, the treatment will not be optimal.

Several of the loop configurations believed to be able to reach desired momentto-force ratios $(M / F)$, and extensively used by orthodontists are vertical helical loop, T-Loop and L-Loop. Siatwoski [5], among others, has studied and designed such loop named as Opus90 and Opus70. Ungbhakorn [6] introduced characteristic analysis theoretically using Castigliano's theorem to evaluate spring stiffness, moment, moment-to-force ratio in many variation of total length and gable. Burstone [7] studied the optimization of anterior and canine retraction. First attempts by the authors on formulating the structural behavior through analytical method were reported using Castigliano method for T-loop $[8,9]$. On the other hand, the development of measurement apparatus as well as measurement attempt on several geometry were also reported [10].

In this paper, the above developed methods are now improved and used extensively to find the relationship between retraction spring geometry and their mechanical behavior, through simulation of a set of samples. Three basic geometries are investigated, namely T-loop, Mushroom, and U-loop. The important mechanical behavior is represented by the force system, consisting of $\mathrm{Fx}, \mathrm{My}$, and $\mathrm{Mz}$ for various activation distance. The influence of each parameter is visualized through graphs, to provide orthodontists with visual description. Furthermore, the database can also be used as the source for knowledge-based design in sectional retraction spring geometry selection using built-in software, which is not discussed in this paper. Interested readers are suggested to refer to Idris, et al. [11,12] and Lim, et al. [13] for the discussion on the geometry selection using knowledge-based design. 


\section{Simulation}

\subsection{Analytical Method}

In order to obtain the analytical solution, an energy method based on the second theorem of Castigliano was used. According to the theorem, in an elastic body that is sufficiently supported and subjected to a number of external loads, it will generally deform under the action of the forces, causing them to do work which is stored as strain energy. For beam mainly under flexural deformation, the strain energy may be expressed as follows.

$$
U=\frac{1}{2 E I} \int M_{n} d l
$$

where $M_{n}$ represents moment appllied to each beam caused by external forces and moments applied to the retraction spring, and $E I$ is combined flexural stiffness of each beam.

The second theorem of Castigliano was used as follows [14],

$$
u_{i}=\frac{\partial U\left(F_{i}\right)}{\partial F_{i}}
$$

where $u_{i}$ are the displacement, $F_{i}$ are the corresponding external force, $F_{x}$ or moment, $M_{z}$; and strain energy $U$, is expressed in that external force or moment.

In this case, the retraction spring is loaded in displacement, with a certain activation distance, $u_{x}$, and angle, $\theta$. Therefore, the above general equation may be expressed with respect to that activation distance and angle respectively. Detail and results of derivation could be found in [8] and [9].

\subsection{Finite Element Method}

Finite element analysis is carried out using ANSYS Multiphysics 11.0 SP1 general purpose FE software. It uses an explicit analysis option available within the software for realistic modeling. For the model geometry, line model are used to simplify the calculating process, without sacrificing the accuracy. Beam elements were chosen, namely BEAM4, BEAM44, and BEAM188. BEAM44 and BEAM188 have the capability of dynamic analysis, thus it will make the calculating process longer or inefficient for quasi-static analysis as in the retraction spring application. Therefore, it may be concluded that BEAM4 is the most suitable element type for this case. When the spring is being activated, the wire undergoes large deformation. This large deformation will result in stiffness 
change that is caused by element shape and orientation changing (stress stiffening effects). These effects result in nonlinearity of forces and moments produced by the activated spring; hence non-linearity behavior analysis is also utilized.

In order to obtain accurate numerical model, two simulation models were attempted. The first was a model with fixed constraints on each spring end, whilst the second was with three-point constraint on each spring end (Figure 4). The fixed constraint model is simpler and commonly used, while the three-point constraint model is considered closer to the actual contact condition between the spring and bracket.

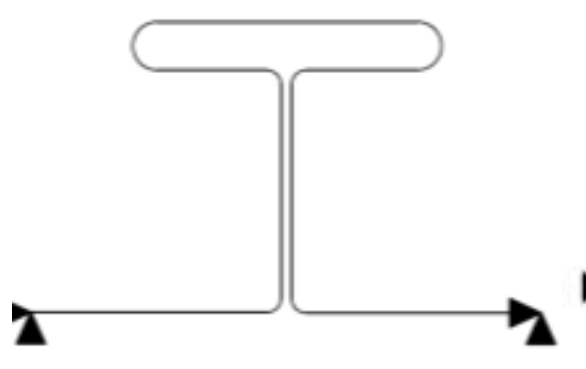

(a)

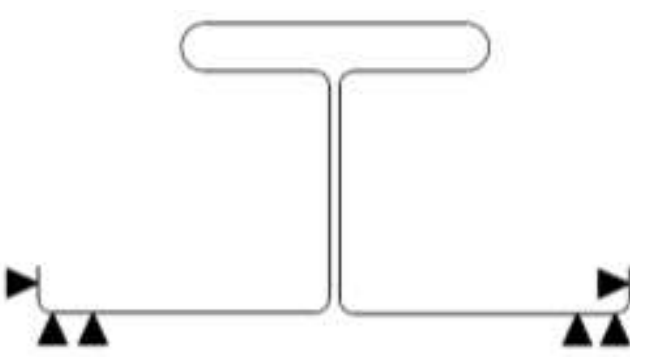

(b)

Figure 4 Alternative simulation model of constraint on brackets: a) Fixed constraint, b) three-point constraint.

\subsection{Validation}

Model validation was conducted to compare numerical (FEM) result with that of analytical. Three basic geometry of retraction spring have been evaluated, as shown in Table 1.

The main assumption of the theoretical analysis is linear-elastic for material model, and remains on elastic range after activation is applied. Other assumptions include: small deformation, the plane cross section of wire remains plane after deformation or shape distortion is negligible after activation. The assumptions preclude the ability to simulate geometrical non-linearity. In reality, when a large deformation is applied, nonlinearity is expected to occur on force system. Numerical method, though, is expected to be able to resolve the problem. The reaction force, $F_{x}$, and moment, $M_{z}$, are obtained as analysis result. 
Table 1 Three basic geometry of retraction spring.

\begin{tabular}{crcc}
\hline Variable & T-loop & Mushroom & U-loop \\
\hline L1 & $4 \mathrm{~mm}$ & $5 \mathrm{~mm}$ & $5 \mathrm{~mm}$ \\
L2 & $4.5 \mathrm{~mm}$ & $4 \mathrm{~mm}$ & $3 \mathrm{~mm}$ \\
L3 & $3 \mathrm{~mm}$ & $3 \mathrm{~mm}$ & $3 \mathrm{~mm}$ \\
L4 & $8 \mathrm{~mm}$ & $3 \mathrm{~mm}$ & $5 \mathrm{~mm}$ \\
L5 & $3 \mathrm{~mm}$ & $4 \mathrm{~mm}$ & - \\
L6 & $4.5 \mathrm{~mm}$ & $5 \mathrm{~mm}$ & - \\
L7 & $4 \mathrm{~mm}$ & - & - \\
R & $1 \mathrm{~mm}$ & $5 \mathrm{~mm}$ & $1 \mathrm{~mm}$ \\
Gable & $0^{\circ}$ & $0^{\circ}$ & $0^{\circ}$ \\
\hline
\end{tabular}

\section{Geometry}

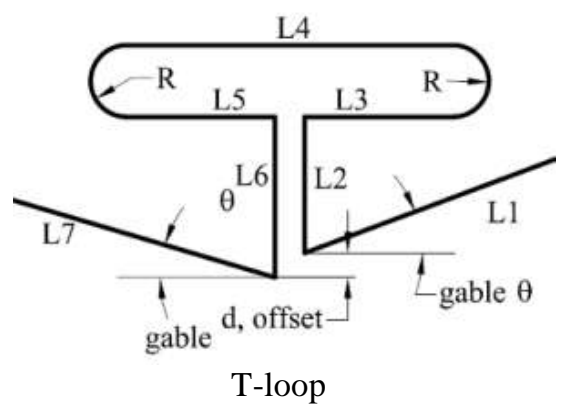

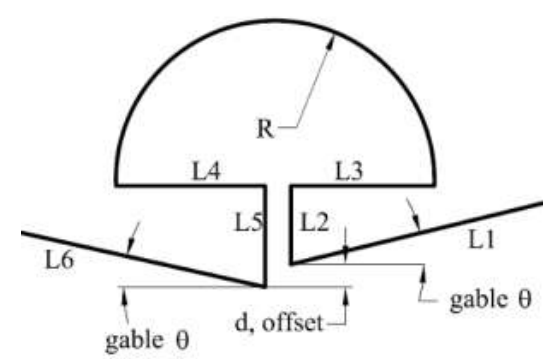

Mushroom

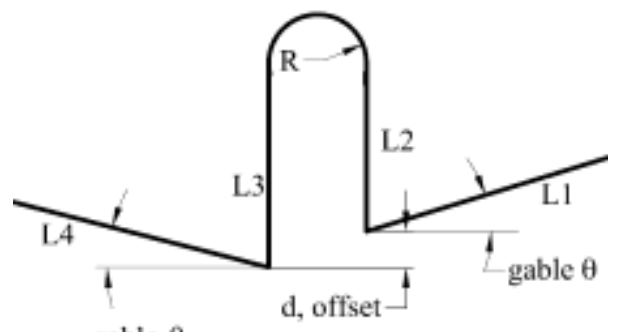

gable $\theta$

U-Loop

Material

Modulus Elasticity, E

Width, B

Height, $\mathrm{H}$

Cross Section Area, A

Moment of Inertia, I
SS, Stainless Steel

$210^{11} \mathrm{~N} / \mathrm{m}^{2}$

$0.5588 \mathrm{~mm}$

$0.4046 \mathrm{~mm}$

$2.310^{-9} \mathrm{~m}^{2}$

$3.110^{-15} \mathrm{~m}^{4}$ 
Table 2 shows the resulting $F_{x}$ and $M_{z}$ obtained by theoretical, linear FEM and nonlinear FEM computation for the three basic geometries evaluated. Theoretical and FEM (linear) results have little different in force and moment, as predicted, since both methods apply a linear assumption. The linearity of force and moment results in constant value of moment-to-force ratio $(M / F)$. Contrary to non-linear analysis, force and moment have nonlinearity in larger deformation. This results in non-constant result of moment-to-force ratio (decreasing to activation). This non-linear approach is expected to be closer to the real large-deformation condition.

Table 2 Result comparison of T-loop, Mushroom and U-loop.

\begin{tabular}{|c|c|c|c|c|c|c|c|c|c|}
\hline \multicolumn{10}{|c|}{ T-loop } \\
\hline & \multicolumn{3}{|c|}{ Theoretical } & \multicolumn{3}{|c|}{ FEM (Linear Analysis) } & \multicolumn{3}{|c|}{$\begin{array}{c}\text { FEM (Non-Linear } \\
\text { Analysis) }\end{array}$} \\
\hline $\begin{array}{c}u_{x} \\
(\mathrm{~mm})\end{array}$ & $\begin{array}{c}F_{x} \\
(\text { grf) }\end{array}$ & $\begin{array}{c}\quad M_{z} \\
(\mathrm{grf} . \mathrm{mm})\end{array}$ & $\begin{array}{l}M_{z} / F_{x} \\
(\mathrm{~mm})\end{array}$ & $\begin{array}{l}F_{x} \\
\text { (grf) }\end{array}$ & $\begin{array}{c}\quad M_{z} \\
\text { (grf.mm) }\end{array}$ & $\begin{array}{l}M_{z} / F_{x} \\
(\mathrm{~mm})\end{array}$ & $\begin{array}{l}F_{x} \\
(\text { grf })\end{array}$ & $\begin{array}{c}M_{z} \\
\text { (grf.mm) }\end{array}$ & $\begin{array}{l}M_{z} / F_{x} \\
(\mathrm{~mm})\end{array}$ \\
\hline 0.00 & 0 & 0 & - & 0 & 0 & - & 0 & 0 & - \\
\hline 1.00 & 268 & 962 & 3.59 & 271 & 981 & 3.62 & 265 & 958 & 3.62 \\
\hline 2.00 & 536 & 1924 & 3.59 & 542 & 1962 & 3.62 & 513 & 1848 & 3.61 \\
\hline \multicolumn{10}{|c|}{ Mushroom } \\
\hline & \multicolumn{3}{|c|}{ Theoretical } & \multicolumn{3}{|c|}{ FEM (Linear Analysis) } & \multicolumn{3}{|c|}{$\begin{array}{c}\text { FEM (Non-Linear } \\
\text { Analysis) }\end{array}$} \\
\hline $\begin{array}{c}u_{x} \\
(\mathrm{~mm})\end{array}$ & $\begin{array}{l}F_{x} \\
\text { (grf) }\end{array}$ & $\begin{array}{c}M_{z} \\
(\mathrm{grf} . \mathrm{mm})\end{array}$ & $\begin{array}{l}M_{z} / F_{x} \\
(\mathrm{~mm})\end{array}$ & $\begin{array}{l}F_{x} \\
\text { (grf) }\end{array}$ & $\begin{array}{c}\quad M_{z} \\
\text { (grf.mm) }\end{array}$ & $\begin{array}{l}M_{z} / F_{x} \\
(\mathrm{~mm})\end{array}$ & $\begin{array}{l}F_{x} \\
(\mathrm{grf})\end{array}$ & $\begin{array}{c}\quad M_{z} \\
\text { (grf.mm) }\end{array}$ & $\begin{array}{l}M_{z} / F_{x} \\
(\mathrm{~mm})\end{array}$ \\
\hline 0.00 & 0 & 0 & - & 0 & 0.00 & - & 0 & 0 & - \\
\hline 1.00 & 157 & 604 & 3.85 & 158 & 619 & 3.88 & 159 & 619 & 3.84 \\
\hline 2.00 & 314 & 1209 & 3.85 & 317 & 1230 & 3.88 & 318 & 1205 & 3.79 \\
\hline \multicolumn{10}{|c|}{ U-Loop } \\
\hline & \multicolumn{3}{|c|}{ Theoretical } & \multicolumn{3}{|c|}{ FEM (Linear Analysis) } & \multicolumn{3}{|c|}{$\begin{array}{c}\text { FEM (Non-Linear } \\
\text { Analysis) }\end{array}$} \\
\hline $\begin{array}{c}u_{x} \\
(\mathrm{~mm})\end{array}$ & $\begin{array}{c}F_{x} \\
(\text { grf) }\end{array}$ & $\begin{array}{c}\quad M_{z} \\
\text { (grf.mm) }\end{array}$ & $\begin{array}{l}M_{z} / F_{x} \\
(\mathrm{~mm})\end{array}$ & $\begin{array}{c}F_{x} \\
(\text { grf })\end{array}$ & $\begin{array}{c}M_{z} \\
\text { (grf.mm) }\end{array}$ & $\begin{array}{l}M_{z} / F_{x} \\
(\mathrm{~mm})\end{array}$ & $\begin{array}{l}F_{x} \\
(\text { grf })\end{array}$ & $\begin{array}{c}M_{z} \\
\text { (grf.mm) }\end{array}$ & $\begin{array}{l}M_{z} / F_{x} \\
(\mathrm{~mm})\end{array}$ \\
\hline 0.00 & 0.00 & 0.00 & - & 0 & 0 & - & 0 & 0 & - \\
\hline 0.50 & 821 & 877 & 1.07 & 811 & 883 & 1.09 & 88 & 860 & 0.97 \\
\hline 1.00 & 1643 & 1753 & 1.07 & 1622 & 1760 & 1.09 & 1883 & 1615 & 0.86 \\
\hline
\end{tabular}


Table 3 Dimension geometry comparison between specimens and FEM.

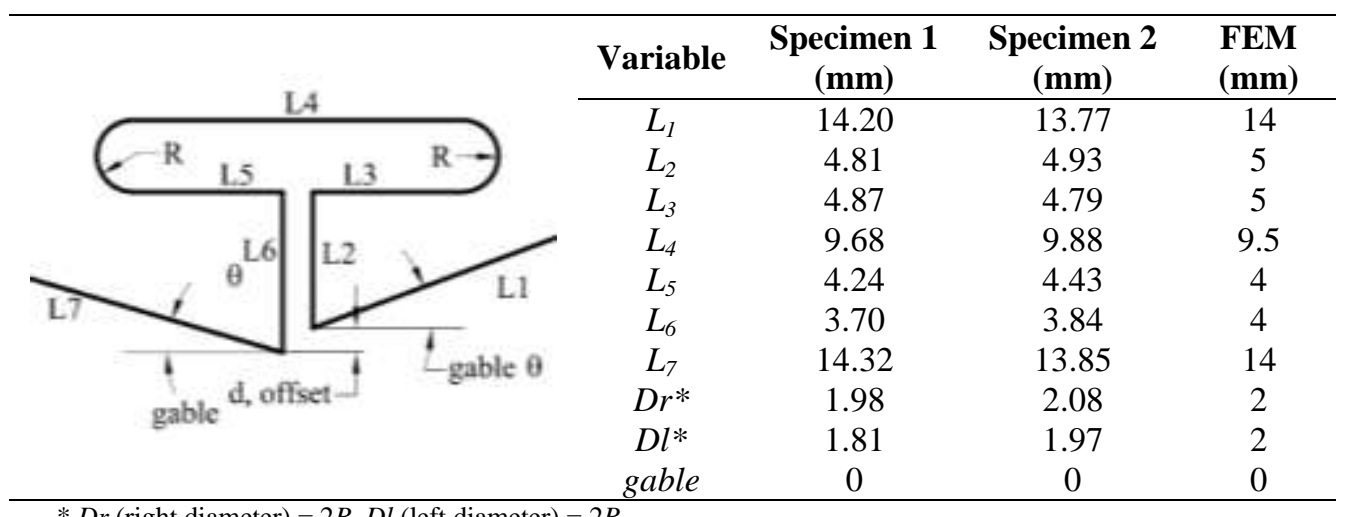

* $D r$ (right diameter $)=2 R, D l$ (left diameter $)=2 R$
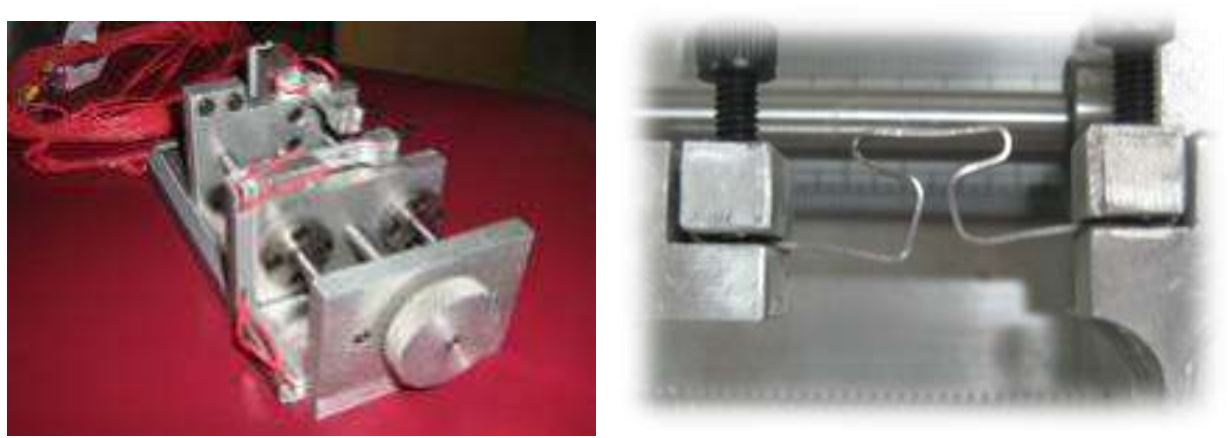

Figure 5 Experimental apparatus for measuring force and moment of a retraction spring [10].

Numerical analysis result (FEM) was also validated against experimental results of two identical specimens of T-loop with TMA material and $0.016 \times 0.022$ in of cross section. Detail geometry of T-loop being tested is shown in Table 3. There are discrepancies between the dimensions of the two actual specimens and the desired dimensions, which is used in the FE analysis. This was due to inaccuracies in manual production of the spring. The experiment used an inhouse-built apparatus capable of measuring both force and moment of a retraction spring, as shown in Figure 5. In the experimental apparatus, both legs of retraction spring are fixed on the jig, whereas one of the jig is movable to simulate activation distance. This pair of jigs also serves as a combined force and moment sensor. 


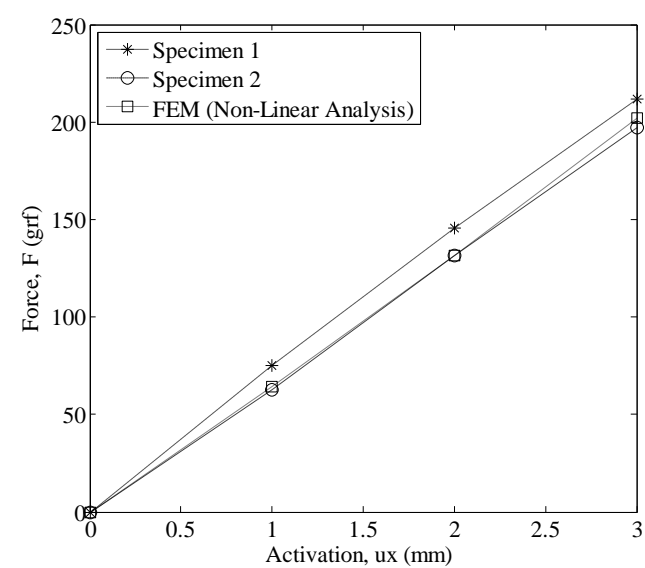

(a)

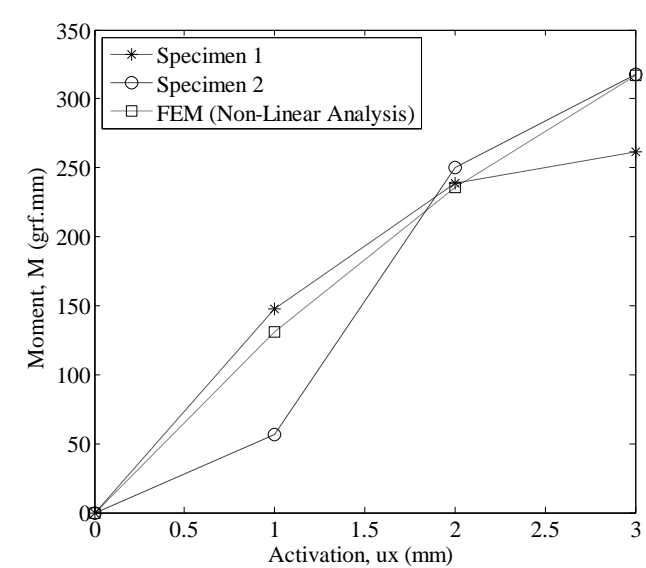

(b)

Figure 6 Comparison result between numerical and experimental method of 2 T-loop specimens: (a) Force against activation and (b) Moment against activation.

Detail description of the development and performance assessment of the abovementioned measuring device is reported by Setiawan [10]. The comparison between experimental and the non-linear FE simulation result are presented in Figure 6. Two specimens have been used for this comparison.

The FEA results agree reasonably well with the experiments, especially on the force result, whilst less was found in the moment case, with the maximum error of $30 \%$ (against specimen 2 at $1 \mathrm{~mm}$ activation). Figure 6 shows that there is considerable doubt in the measurement result on the worst comparison result that is not shown with specimen 1 . In specimen 1 , it is found that the tendency of non-linear behavior is predicted sufficiently well with the FEM. Imperfectness in the measurement devices could also contribute to the discrepancies, especially in the moment measurement. As discussed in [10], the fixation of the wire to the bracket plays important role in the moment produced, hence discrepancies in the moment comparison with the idealized model in FE analysis. It is expected that during the clinical application of such retraction spring would introduce similar, if not, higher uncertainty as in the experiments. This remain another subject of discussion in order to understand the behaviour of the spring during application.

Apart from owned experiment, the validation of the numerical method was carried out with the experimental result by Chen [2]. In the report, stainless steel wire of $0.016 \times 0.022$ cross section $(0.4064 \mathrm{~mm} \times 0.5588 \mathrm{~mm})$ was used, as shown in Figure 7. 


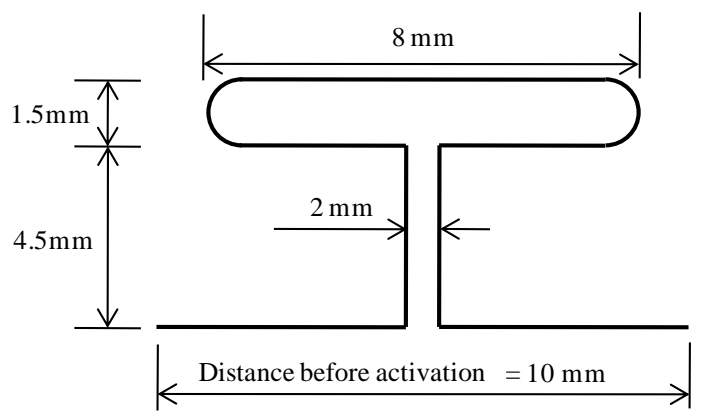

Figure 7 Specimen dimension by Chen [2].

As shown in Table 4, the result in force gives a good agreement between Chen's and FEM. However, as in the previous comparison, FEM results underestimate the moment calculation by up to $24.88 \%$. It is suggested that similar explanation is applied to this case, i.e. the fixation model of the wire on the bracket. The slight differences on the dimension are considered not significant to cause such high error in moment.

By comparing the two FEA results against experiments, it is concluded that the FE model provides a sufficient prediction on the force, whilst less in moment. This is believed to be caused by uncertainties in the fixation of the wire to the bracket during the experiments. This problem is thought to be worse in the application of the retraction spring on the actual tooth. Nevertheless, due to very good estimation on the force, the current FE model was used to generate database in order to obtain a more comprehensive mechanical behavior of the retraction spring, as shown in Table 4 and Figure 8.

Table 4 Comparison force and moment between Chen [2] and FEM.

\begin{tabular}{cccc}
\hline Activation & Chen & FEM & Error \\
\hline $\boldsymbol{u}_{\boldsymbol{x}}(\mathbf{m m})$ & \multicolumn{2}{c}{ Force, $\boldsymbol{F}$ (grf) } & $\mathbf{( \% )}$ \\
\hline 0 & 0 & 0 & \\
1 & 500 & 493.40 & 1.32 \\
2 & 980 & 972.06 & 0.81 \\
\hline$(\mathbf{m m})$ & Moment, $\boldsymbol{M}$ (grf.mm) & $\mathbf{( \% )}$ \\
\hline 0 & 0 & 0 & \\
1 & 1250 & 1560.96 & 24.88 \\
2 & 2490 & 3014.31 & 21.06 \\
\hline
\end{tabular}




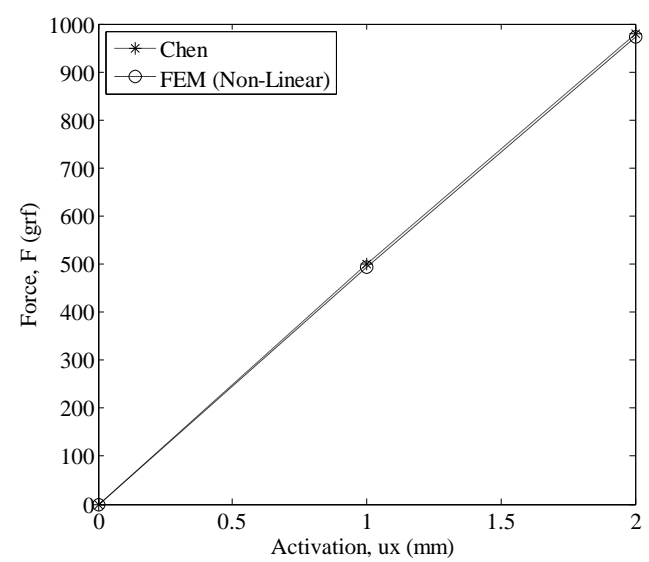

(a)

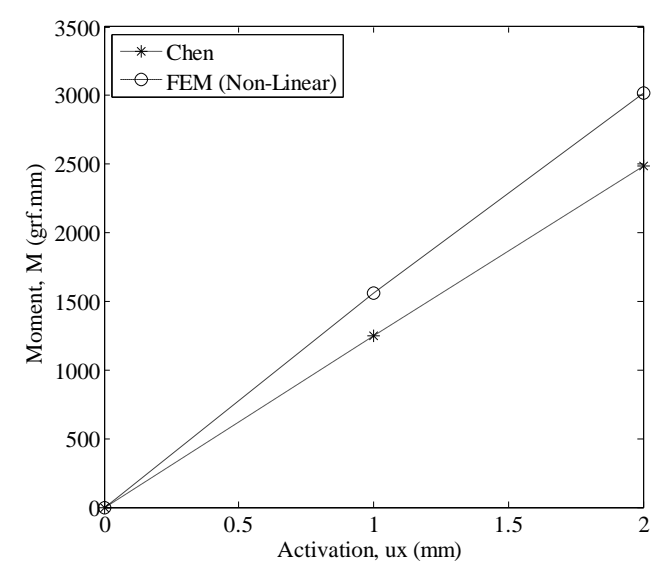

(b)

Figure 8 Comparison of force and moment between FEM and experiment by Chen [2].

\section{Mechanical Behavior}

In order to provide comprehensive understanding of the mechanical behavior of orthodontic retraction spring, simulation on a number of samples was carried out. The three basic geometries as previously discussed were used, i.e. T-loop, Mushroom, and U-loop, with the geometry parameters to be considered are shown in Table 3. The mechanical behavior relates dimensions as input parameters or design variables with the force system, consisting of $F_{x}, M_{y}$, and $M_{z}$, as the output parameters. The result is then presented in 3D graphs, visualizing the influence of important parameters to each output parameters. The surface visualization of the response was generated using GRIDDATA function available in MATLAB. The two important parameters are chosen over the initial input parameters in order to provide an easier-to-use graphs for the orthodontists. The result presented is for the case of $2 \mathrm{~mm}$ activation distance, which represents mid-distance of normal activation in the application. The material used in the simulation is stainless steel with the modulus of elasticity of approximately $200 \mathrm{GPa}$. The range of dimensions is set by considering the design space for geometry between two teeth, as well as the manual method of production.

\subsection{T-loop}

T-loop is one of the most common forms of retraction spring used for orthodontic application. It is commonly used for overcoming uncontrolled movement due to force and moment. Characteristics of force and moment may 
be explained in two input parameters, i.e. total height and leg total length of the design. As shown in Figure 9, loop total height has more significant influence to force compared with the leg total length. Its force decreases quadratically due to increasing the total height parameter. For moment, smaller total height and leg total length parameter will produce higher moment, quadratically. Momentto-force ratio seems to be much more sensitive to total height than to leg total length. Leg total length plays important role to moment if combined with low total height.

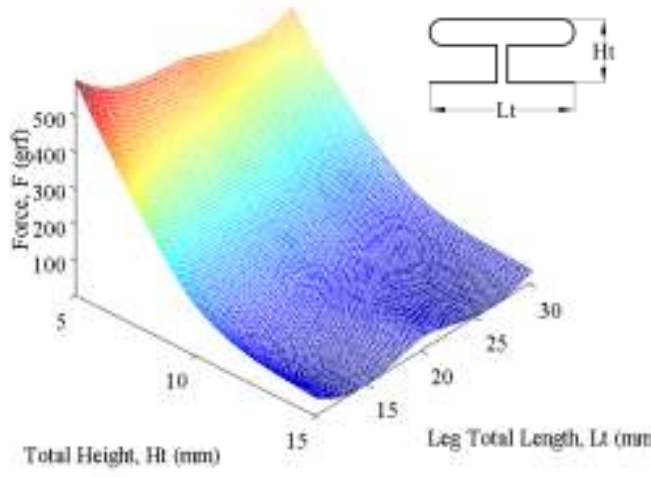

(a)

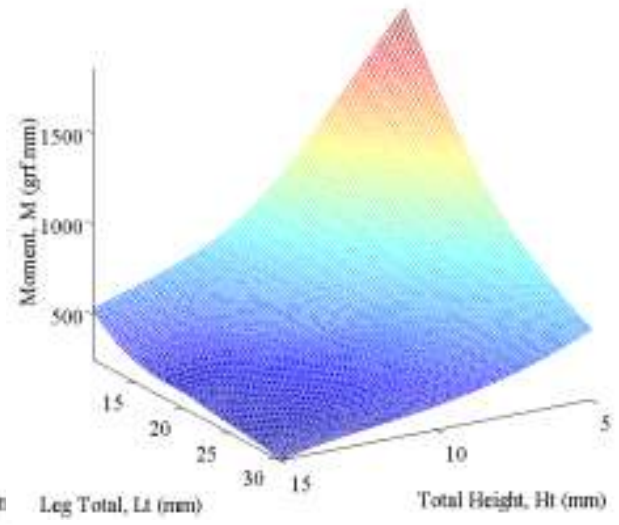

(b)

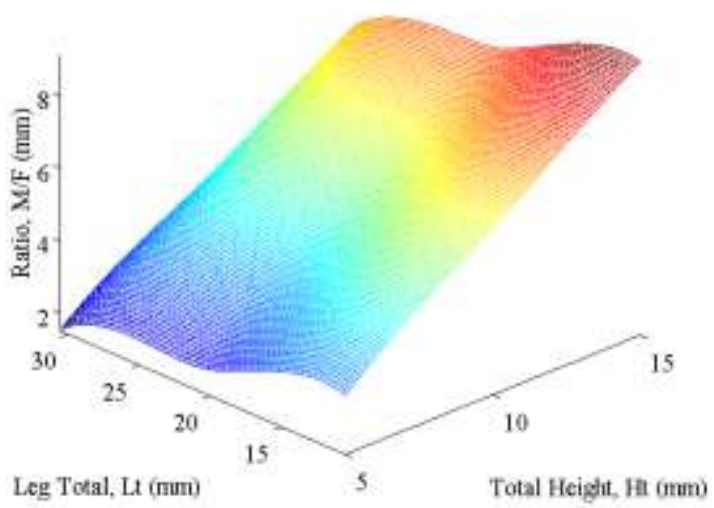

(c)

Figure 9 Influence of Leg total length and Total height for T-loop type: a) Force, b) Moment, b) Moment-to-force ratio.

\subsection{Mushroom}

The characteristics of force and moment produced by mushroom loop have similar tendency to T-loop design. Characteristic force and moment can also be 
explained in two input parameters such as, total height and leg total length of design. Total height parameter has more significant influence to force, compared with the leg total length. Mushroom loop produces relatively higher force and moment than T-loop design does, for similar total height and leg total length.

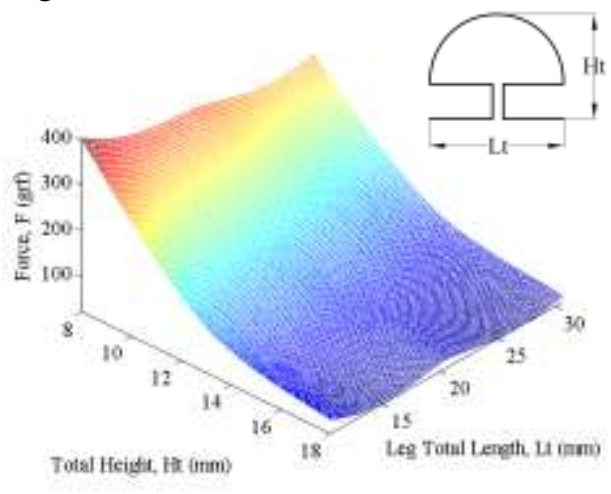

(a)

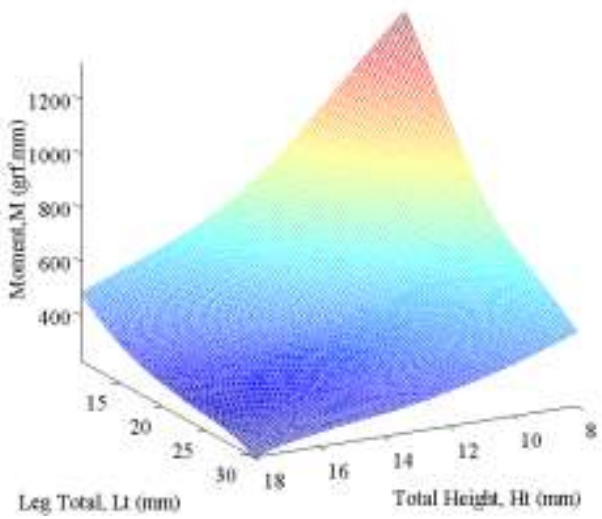

(b)

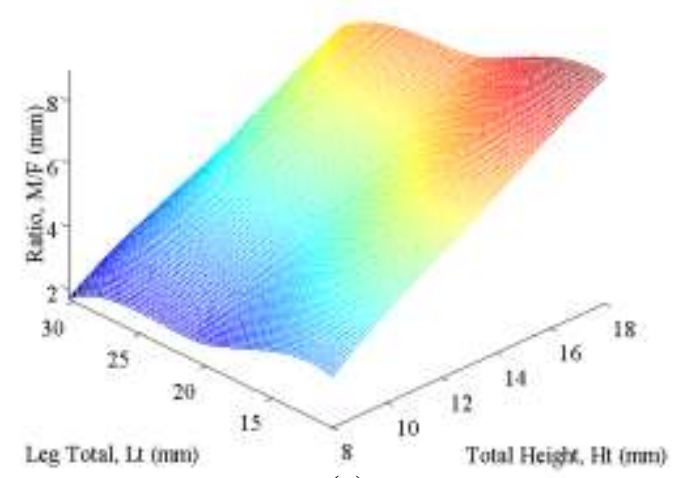

(c)

Figure 10 Influence of Leg total length and Total height for Mushroom-type: a) Force, b) Moment, b) Moment-to-force ratio.

\subsection{U-loop}

U-loop is one of the simplest forms of retraction spring used for orthodontic application. U-loop is one of design that overcomes movement due to big force and moment. Force will decrease quadratically due to increasing the total height parameter. Furthermore, for total height of more than $12 \mathrm{~mm}$, the change in force is insignificant. This could indicate an upper bound of U-loop design. On the other hand, shorter loop total height has a significant influence in the force. In fact, for a height of $10 \mathrm{~mm}$ or more, the U-loop produces too high (of a) force 
for a tooth. This also could be taken as a lower-bound of U-loop design. Smaller total height and leg total length parameter produces higher moment, quadratically. M/F ratio is highly influenced by the total height parameter, and increases linearly with increasing total height.

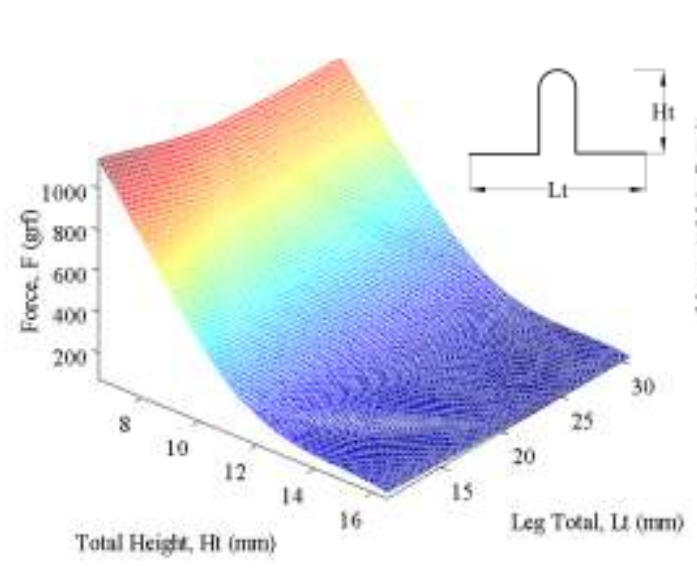

(a)

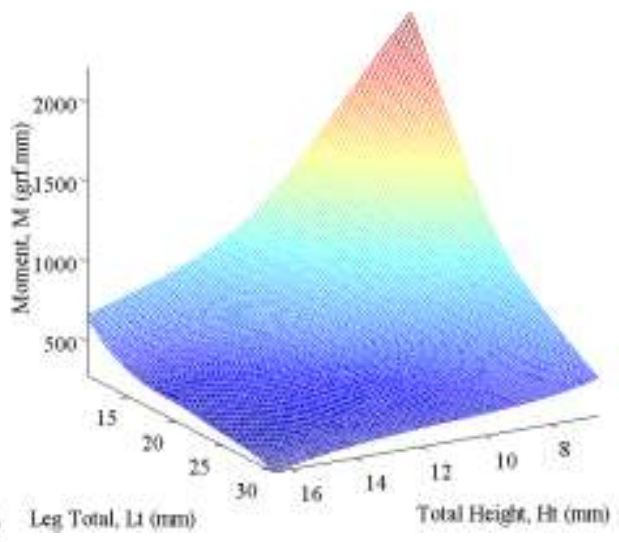

(b)

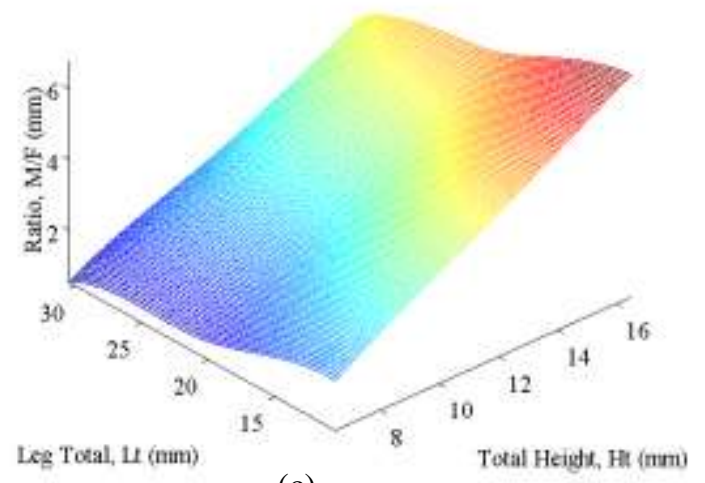

(c)

Figure 11 Influence of Leg total length and Total height for U-loop type: a) Force, b) Moment, c) Moment-to-force ratio.

\section{Discussion}

From the 3D visualization, it may be seen that the three loop design have similar characteristics with respect to leg total length and total height. The difference is the range of force system produced. U-loop tends to be stiffer than T-loop, hence produces higher force for the same activation distance, whereas mushroom-type has lower stiffness. It also provides large combination of 
moment-to-force ratio. Furthermore, from quantitative analysis described above, a summary of influence of each parameter to force, moment, and moment-toforce ratio is presented in Table 5. This summary can be used as guidance for orthodontists during the selection of orthodontic wire dimensions.

Table 5 Summary of general influence of important geometry to force system.

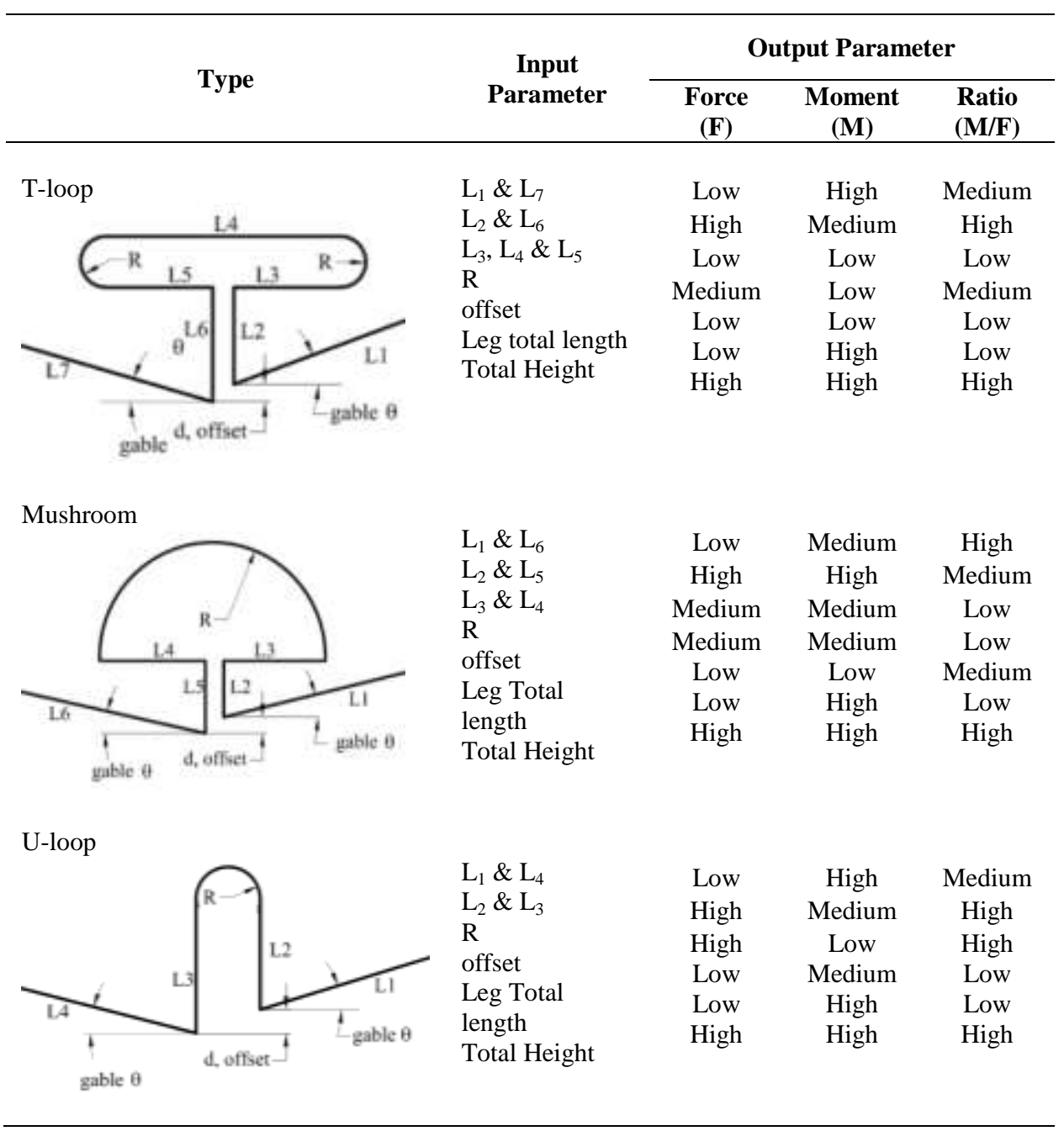

Comparing the 3 loop design, it can be seen that U-loop gives the most sensitive response of total leg length to the force, whilst T-loop gives the least sensitive. In all models, the smaller the overall size of the loop, the higher is the resulting moment. The total leg length gives a less significant influence towards the force, whilst the total height is a significant parameter as the force is very sensitive to the total height. Among the three models, the total length of T-loop 
gives the least significant, whilst U-loop gives the most significant effect to the force. T-loop provides the lowest relative force, whereas U-loop provides the highest force, i.e. up to 1000 grf in smaller total height. Using softer material, such as TMA with the modulus of elasticity of $79 \mathrm{GPa}$, the value of force could be down to approximately 400 grf. In fact this value is considered too high for orthodontic application, since the optimum force for normal application is only up to $120 \mathrm{grf}$ [3]. U-loop has relatively lower moment-to-force ratio among the other two models. This ratio is important to provide a control in movement. Too low ratio could result in rotation of tooth movement instead of bodily or translation. Furthermore, in theory, the cross-section and material type affect both force and moment proportionally to the modulus of elasticity, whilst gives no influence in the moment-to-force ratio. However, the non-linear behavior of other materials, e.g. TMA and $\mathrm{NiTi}$, need to be investigated further experimentally, as simulation could have a significant discrepancies for these materials.

\section{Conclusions}

In order to understand the characteristics of several geometry of orthodontic wire, theoretical as well as finite element method (FEM) have been used. Theoretical solution provides linear relationship between activation distance and force and moment, whilst FEM can simulate geometry non-linearity that exists in reality. Finite element model provides accurate prediction of forces, whilst overestimate the moments when compared with in-house experimental results as well as experiments done by other researchers. With FEM, mechanical behavior of 3 basic geometry of orthodontic spring is simulated for a number of combinations of parameters.

Table 5 is presented as a basic reference to understanding the behaviour of three basic geometries. The knowledge may be used as guidance for geometry selection in orthodontic application. The next research stage is enriching the design database with other geometry, then apply it for a-more-accurate geometry selection using knowledge-based design methodology, as well as more user-friendly.

\section{Acknowledgements}

The research was funded by the State Ministry of Research and Technology, Republic of Indonesia, under the contract No. 53/RT/Insentif/PPK/II/2008 (year I), and No. 050/RT/D.PSIPTN/PPK/I/2009 (year II), for which the authors express their gratitude. 


\section{References}

[1] Nanda, R., Biomechanics in Clinical Orthodontic, W.B. Sauders Company: Phildelphia, pp.156-187, 1997.

[2] Chen, J., D.L. Markham \& T.R. Katona, Effect of T-Loop Geometry on It's Forces and Moments. The Angle Orthodontist, 70, pp. 48-51, 2000.

[3] Funk \& C. Albert, The Heat-Treatment of Stainless Steel. The Angle Orthodontist, 21, pp. 129-138, 1951.

[4] Kapila, S. \& R. Sachdeva, Mechanical Properties and clinical Applications of Orthodontic Wires, American Journal of Orthodontics and Dentofacial Orthopedics, 96, pp. 100-109, 1989.

[5] Siatkowski, R.E., Continuous Arch Wire Closing Loop Design, Optimization and Verification. Part L Am J Orthod Dentofac Orthop, 112, p. 393-402, 1997.

[6] Ungbhakorn, V., V. Ungbhakorn \& P. Techalertpaisarn, Assessment of Castigliano's Theorem on The Analysis of Closing Loop for Canine Retraction by Experiment and Finite Element Method Part I. Thammasat Int.J . Sc.Tech., 10, pp. 28-37, 2005.

[7] Burstone, C. \& H. Koenig, Optimizing Anterior and Canine Retraction. American Journal of Orthodontics and Dentofacial Orthopedics, 70, pp. 1-19, 1976.

[8] Putra, B., Geometry Selection of Sectional Retraction Spring using Knowledge-based Design, Institut Teknologi Bandung: Bandung, 2008.

[9] Setiawan, R., Putra, B.B. \& Wachyudi, N., Geometry Selection of Orthodontic Retraction Spring Through Knowledge-based design. in Joint Meeting JSME ch. Indonesia - AUN/SEED Net Regional Workshop, Bandung, Indonesia: AUN/SEED Net, 2008.

[10] Setiawan, R., Yudha, L.P. \& Wibowo, A., Design, Manufacturing, and Testing of 3-Axis Force System Orthodontic Springs, in SNTTM VIII, Semarang, 2009.

[11] Idris, M., et al., Geometry Selection of Orthodontic Retraction Spring Using Global Optimization Method. in Regional Conference on Mechanical and Aerospace Technology, Bali, Indonesia, 2010.

[12] Idris, M., Optimization for Geometry Selection of Sectional Retraction Spring Using Memetic Algorithm with Design Database, Institut Teknologi Bandung, 2010.

[13] Lim, D., et al. Classifier-assisted Constrained Evolutionary Optimization for Automated Geometry Selection of Orthodontic Retraction Spring. in 2010 IEEE Congress on Evolutionary Computation Proceeding, Barcelona, 2010.

[14] Den Hartog, J.P., Advanced Strength of Material, Nevada: Dover Publications, 1987. 